\title{
Magnetomotive and Tension-Based Tuning of a Micromechanical Resonator ${ }^{\dagger}$
}

\author{
Mahmut Bicer ${ }^{1}$, Arda D. Yalcinkaya ${ }^{2}$ and B. Erdem Alaca ${ }^{1,3, *}$ \\ 1 Department of Mechanical Engineering, Koc University, Istanbul 34450, Turkey; mbicer13@ku.edu.tr \\ 2 Department of Electrical and Electronics Engineering, Bogazici University, Istanbul 34342, Turkey; \\ arda.yalcinkaya@boun.edu.tr \\ 3 Surface Science and Technology Center, Koc University, Istanbul 34450, Turkey \\ * Correspondence: ealaca@ku.edu.tr; Tel.: +90-212-338-1727 \\ † Presented at the Eurosensors 2017 Conference, Paris, France, 3-6 September 2017.
}

Published: 23 August 2017

\begin{abstract}
Bidirectional frequency tuning in microresonators is demonstrated through the simultaneous use of both mechanical (stretching) and magnetomotive approaches. Stretching is employed to increase the resonance frequency, while the magnetomotive method provides actuation and spring softening. The fabrication process is presented for a double-clamped micromechanical nickel resonator. Lorentz Force is used for excitation and flexural deformation of the resonator. A substrate bending method is utilized to introduce uniaxial tension in the resonator. As a result of flexural deformation, up to $13 \%$ decrease in the resonance frequency is achieved. An $8 \%$ increase in the resonance frequency is obtained by the stretching method. The presented study is the first demonstration of the combination of the aforementioned techniques for the bidirectional and wide-range frequency tuning up to $200 \mathrm{kHz}$.
\end{abstract}

Keywords: mechanical resonator; tunable resonator; magnetomotive tuning; micro/nano electromechanical resonators; resonance frequency tuning; RF MEMS; magnetic actuation

\section{Introduction}

Micro and Nano scale size enable mechanical resonators to have high resonance frequency and require low operation power which makes them very suitable for detection, sensing and Radio Frequency (RF) applications. Sensitivity and operation limits of such devices are decided by their resonance frequencies. However, there are many factors that determine the resonance frequency such as geometry, material properties, and other environmental factors, which makes it arduous to obtain the required stability, accuracy and operation range. The requirement of controllable sensitivity, and stability makes frequency tuning crucial for the mechanical resonator. For instance, electrostatic system is used to tune the frequency range of the vibration detection system from 1 to $10 \mathrm{kHz}$ [1]. Several tuning studies reported a usage of tension-based tuning mechanism to increase the resonance frequency and as well as the Quality factor ( $Q$ factor) [2,3]. In addition, numerous studies employed thermal methods to manipulate the resonance frequency through thermal strain that is generated by expansion or shrinkage of mechanical resonator [4]. On the other hand, magnetomotive mechanism is generally used for actuation purposes while the frequency tuning is realized by other mechanisms. For example, magnetomotive actuation was merged with capacitive tuning for nanomechanical resonator with capability of $Q$ factor tuning [5]. The electromagnetic force is utilized for resonance frequency tuning through a constant force acts on the resonator and deforms it [6]. Available techniques introduce frequency shift in one direction. Furthermore, some of the reported studies require an additional excitation mechanism further increasing the complexity of the system and fabrication method. In contrast, this study 
presents the use of two simple mechanisms for the actuation and bidirectional tuning of the resonance frequency.

\section{Materials and Methods}

Fabrication of micromechanical resonator $(\mu \mathrm{MR})$ initiates with silicon dioxide $\left(\mathrm{SiO}_{2}\right)$ coating with PECVD process on a 4"-diameter silicon wafer (Figure 1a). The process is followed by sputter coating of chromium $(\mathrm{Cr})$ and gold $(\mathrm{Au})$ layers with a thickness of 20-nm and 120-nm, respectively (Figure 1b). Photoresist (AZ5214) is deposited with spin coating and mold-patterned with photolithography (Figure 1c). Nickel (Ni), with 4.6- $\mu$ m-thickness, is deposited into the mold-pattern with electroplating process (Figure 1d). Photoresist removal (Figure 1e) and $\mathrm{Au}-\mathrm{Cr}$ etching form the $\mathrm{Ni}$ $\mu \mathrm{MR}$ (Figure 1f), which is released via $\mathrm{SiO}_{2}$ etching (Figure 1g).

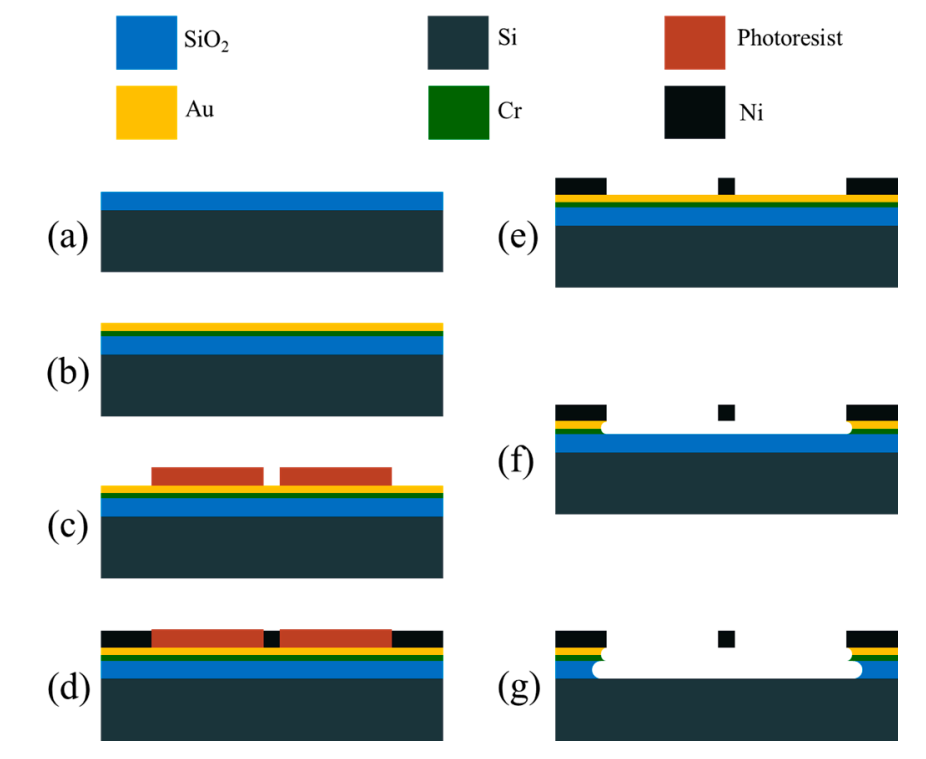

Figure 1. Fabrication process (a) $\mathrm{SiO}_{2}$ coating; (b) $\mathrm{Cr}$ and $\mathrm{Au}$ deposition; (c) Photo-lithography; (d) Electroplating; (e) Photoresist Removal; (f) Au and Cr etching; and (g) Release of Resonator via $\mathrm{SiO}_{2}$ etching.

Suspended $\mu \mathrm{MR}$ has $160 \mu \mathrm{m}$ length, $5 \mu \mathrm{m}$ width and $4.6 \mu \mathrm{m}$ thickness (Figure 2). The bidirectional tuning mechanism and measurement setup are depicted in Figure 3. Figure 3a shows, a clamped-clamped beam, a flexural resonating element, is placed between two permanent magnets which produce a uniform magnetic field. An electrical current with AC and DC components, is led through the $\mu \mathrm{MR}$ in uniform magnetic field. The current passing through the $\mu \mathrm{MR}$ generates a Lorentz force in uniform magnetic field. The AC element of this current excites $\mu \mathrm{MR}$ through a varying Lorentz force while the DC component exerts a constant force on the $\mu \mathrm{MR}$ and give an initial deformation to the beam. An increase in the DC current relatively increases the applied constant force and bending deformation of the resonator which soften the stiffness of $\mu \mathrm{MR}$ thus decreases the resonance frequency. Figure $3 b$ demonstrates the substrate bending method that is employed for realization of the tension-based tuning mechanism. The die contains $\mu \mathrm{MR}$ placed in a holder and a screw is guided through beneath of the holder. The turning of a screw bends the chip, hence induce a tensile stress on the $\mu \mathrm{MR}$ in controlled fashion.

The induced stress hardens the stiffness of $\mu \mathrm{MR}$ and cause an increase in its resonance frequency. All the changes occur on the $\mu \mathrm{MR}$ is captured through variation of its resonance frequency and the resonance peak of the electromechanical transfer function is characterized by the LDV. 


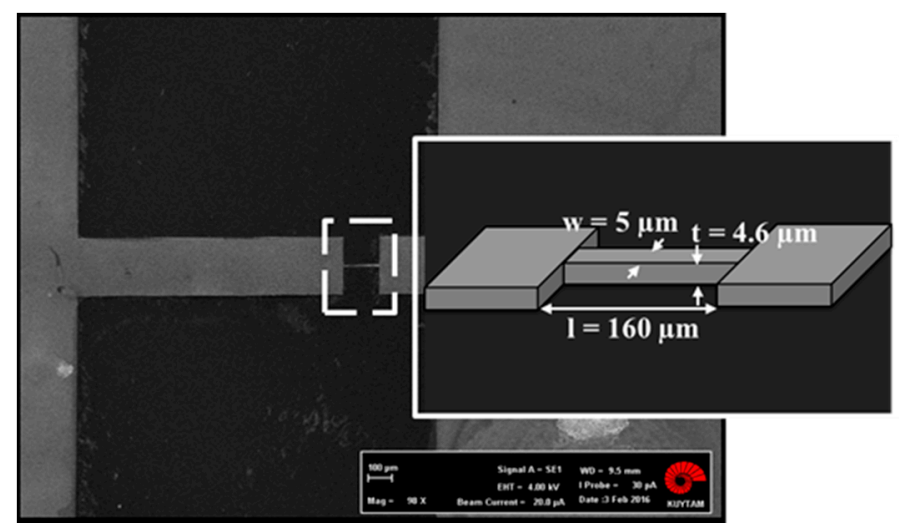

Figure 2. SEM image of Ni microresonator.

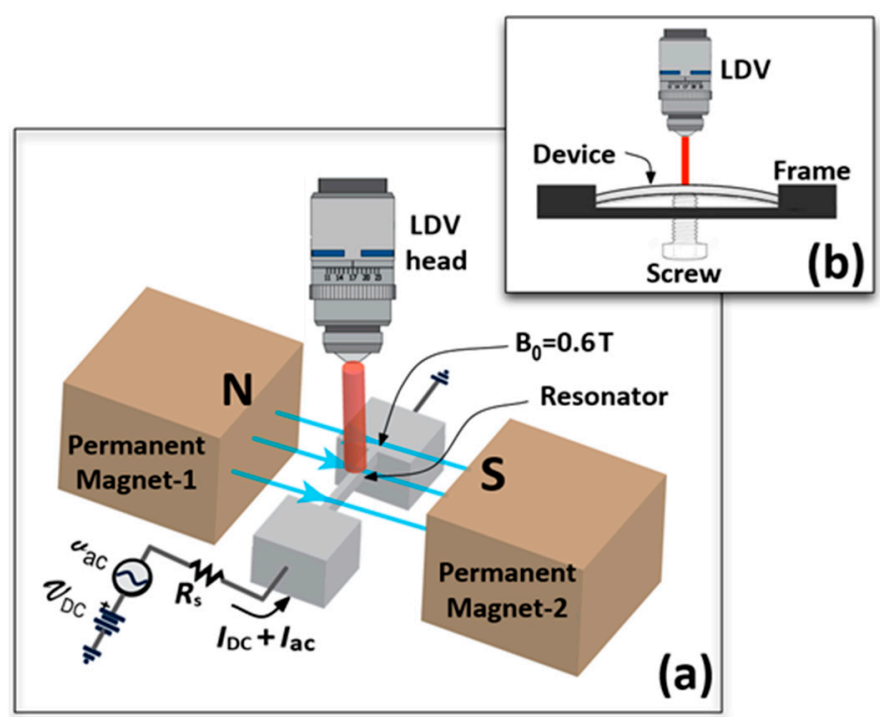

Figure 3. (a) Actuation and Tuning Schematic; (b) Mechanical Loading and Optical Readout Details.

\section{Results and Discussion}

Figure 4 shows the resulting change of the resonance frequency as a function of DC current. Figure 4a depicts a decrease in the resonance frequency from $789 \mathrm{kHz}$ to $683 \mathrm{kHz}$ corresponding to a range of $13 \%$ for the stress-free case. Figure $4 \mathrm{~b}$ demonstrates a maximum of $12 \%$ reduction in the resonance frequency for a 76-MPa axial tensile stress and a frequency modulation between $854 \mathrm{kHz}$ and $751 \mathrm{kHz}$. The reduction in the resonance frequency can be traced back to the $\mu \mathrm{MR}$ deformation under a constant electromagnetic force provided by the DC current. The simultaneous use of both tuning mechanisms provides a 200-kHz tuning range, from $854 \mathrm{kHz}$ to $683 \mathrm{kHz}$. In comparison with previous studies providing frequency tuning in one direction only, the proposed method represents an improvement for bidirectional tuning with a higher tuning range. 

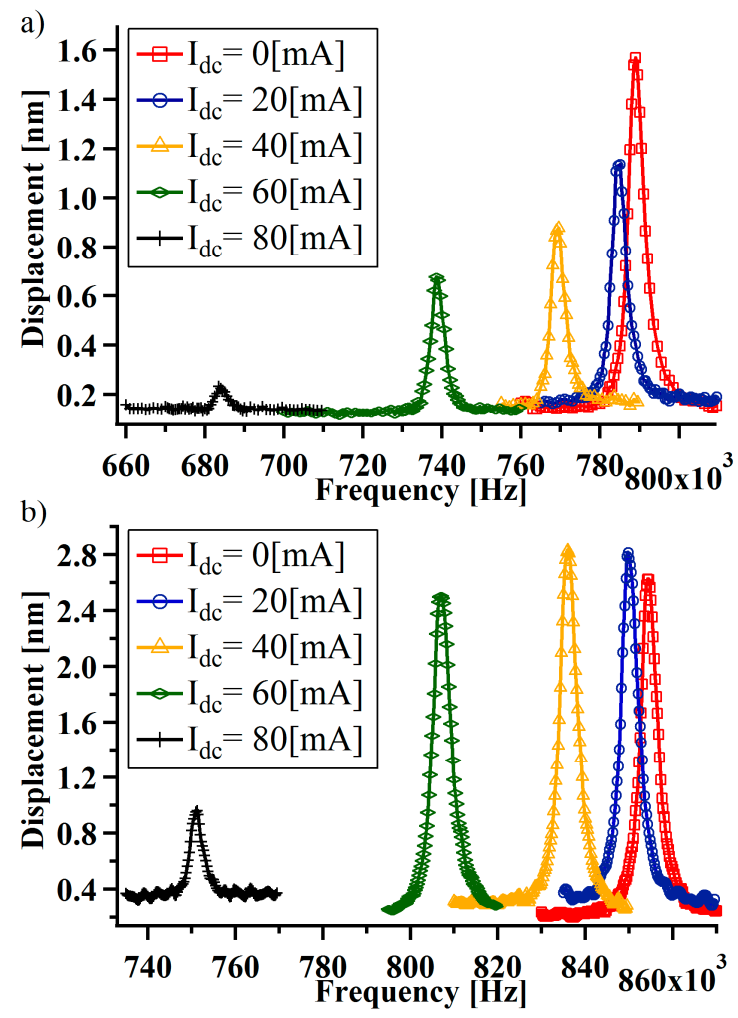

Figure 4. Displacement vs. frequency for applied Idc current for (a) stress-free case; and (b) an applied stress of $76 \mathrm{MPa}$.

Acknowledgments: This work was carried out in Koç University Cleanroom Laboratory supported by ISTKA under Grant TR10/16/YNY/0103 “Nanotechnology Platform for the Accessible and Sustainable Pilot Fabrication of High-Added-Value Products".

Conflicts of Interest: The authors declare no conflict of interest.

\section{References}

1. Scheibner, D.; Mehner, J.; Reuter, D.; Gessner, T.; Dötzel, W. A spectral vibration detection system based on tunable micromechanical resonators. Sens. Actuators A Phys. 2005, 123, 63-72.

2. Verbridge, S.S.; Shapiro, D.F.; Craighead, H.G.; Parpia, J.M. Macroscopic tuning of nanomechanics: Substrate bending for reversible control of frequency and quality factor of nanostring resonators. Nano Lett. 2007, 7, 1728-1735.

3. Ning, Z.Y.; Shi, T.W.; Fu, M.Q.; Guo, Y.; Wei, X.L.; Gao, S.; Chen, Q. Transversally and axially tunable carbon nanotube resonators in situ fabricated and studied inside a scanning electron microscope. Nano Lett. 2014, 14, 1221-1227.

4. Remtema, T.; Lin, L. Active frequency tuning for micro resonators by localized thermal stressing effects. Sens. Actuators A Phys. 2001, 91, 326-332.

5. Dominik, V.S.; Artur, E.; Robert, H.B. Tunable coupled nanomechanical resonators for single-electron transport. New J. Phys. 2002, 4, 86.

6. Ekinci, K.L.; Roukes, M.L. Nanoelectromechanical systems. Rev. Sci. Instrum. 2005, 76, 061101. 